\title{
Lecciones ecológicas de la historia amazónica: impacto diferencial del uso del suelo en las estructuras y biomasas aéreas de bosques secundarios en Napo, Ecuador
}

\author{
Ecological Lessons from Amazonian History: differential impact of land-uses \\ on the structures and aboveground biomass of secondary forests in Napo, Ecuador
}

\author{
Edgardo I Garrido-Pérez a,b*, Katia Laura Sidali c, Jamil Rojas-Salvatierra a, \\ David Tella-Ruíz a,d ${ }^{\text {anngel Cajas }}{ }^{\mathrm{e}}$, María Sol Peña ${ }^{\mathrm{e}}$ \\ *Autor de correspondencia: a Asociació Llapis i llavors (Pencil and seeds), Calle Aragó 565, \\ bajos, Barcelona, España, edgardoga2@hotmail.com \\ ${ }^{b}$ Free University of Bolzano, Faculty-Transgressing project Unlocking the Potential of Social Agriculture in South Tyrol, \\ Brixen, Italia. \\ ${ }^{c}$ Free University of Bozen/Bolzano, School of Economics and Management, Piazzetta dell’Università, 139031 Brunico (BZ), Italia. \\ ${ }^{\mathrm{d}}$ Universitat de Barcelona, Facultat de Geografia i Historia, Departament d'Historia d'America i África, Carrer Montalegre 6-8, \\ Barcelona, España. \\ e Universidad Regional Amazónica -IKIAM, Vía Muyuna, km.7, Tena, Napo, Ecuador.
}

\begin{abstract}
SUMMARY
Amazonian forests mitigate climate change, are being deforested and recover their structures and functions throughout secondary succession. It is necessary to understand how different land-uses alter such a recovery and why landowners decide to clear-cut some areas while letting others to fallow. That was addressed by counting and measuring all trees of at least $10 \mathrm{~cm}$ diameter in four onehectare plots whose land-use histories were exhaustively reconstructed using ethnographic-historical methods. Plots A (12-15 years old), B (22-25 years old) and C (35-37 years old) were pastures and oligocultures for feeding human populations growing during a period of vigorous oil-exploration; next, food demands changed and plots were abandoned. Plot D (35-40 years old) was used for self-consumption, shifting agriculture (more than 50 planted species), afterwards used and managed for extracting resources during secondary succession. Tree diameters in plots B and $\mathrm{C}$ were larger compared to those found in plots A and D -which were similar. The latter suggests that fallow management in plot D kept its forest structurally young, hence, the Amazon may include forests chronologically older than the age suggested by their structures. Similar results were found for total basal area and aboveground biomass, while the high density of individuals in plot D suggests a vigorous gap regeneration. In conclusion: different land-uses are confirmed to alter further succession, and the changes of food demand and food security affect farmer's decisions on letting forests to recover. Local development planners may take the later into account.
\end{abstract}

Key words: carbon dioxide, ethno-ecology, secondary succession, shifting agriculture.

\section{RESUMEN}

Los bosques amazónicos mitigan el cambio climático, sufren deforestación y regeneran su estructura y funciones por sucesión secundaria. Por ello es necesario estudiar cómo distintos usos del suelo alteran dicha recuperación, y por qué se los deforesta o deja regenerar. Para investigarlo, se inventariaron los árboles $($ dap $\geq 10 \mathrm{~cm})$, reconstruyendo las historias de uso de cuatro parcelas de una hectárea mediante métodos etnográfico-históricos. Las parcelas A (12-15 años), B (22-25 años) y C (35-37 años) fueron potreros y oligo-cultivos, para alimentar una creciente población asociada a exploraciones petroleras, luego abandonadas por cambios de demanda alimentaria. La parcela D (35-40 años) fue autárquicamente usada para agricultura itinerante (más de 50 especies), luego para extracción de recursos maderables y no maderables durante la sucesión. Los diámetros arbóreos en $\mathrm{B}$ y C fueron mayores que en A y D, siendo estas últimas semejantes entre sí. El predominio de árboles delgados en D y A, sugiere que el manejo durante la sucesión "rejuveneció" al bosque en D y que en la Amazonía puede haber bosques más viejos que lo indicado por los diámetros de sus árboles. Algo similar ocurrió para el área basal total y la biomasa aérea, aunque la alta densidad de individuos en D sugiere una vigorosa regeneración de los claros. En conclusión, los distintos usos del suelo alteran la sucesión, los cambios de la demanda y seguridad alimentarias afectan la decisión campesina de permitir que las selvas se regeneren, y la gestión territorial puede optimizarse tomando lo antedicho en consideración.

Palabras clave: agricultura itinerante, dióxido de carbono, etno-ecología, sucesión secundaria. 


\section{INTRODUCCIÓN}

Dos aspectos conocidos de los bosques amazónicos son: su mitigación del cambio climático al capturar dióxido de carbono en la biomasa (Brienen et al. 2015), y su alta tasa de deforestación (e.g. un $29 \%$ entre 2015 y 2016 con respecto al periodo anterior en Brasil) (INPE 2016). Pero también ocurren procesos de recuperación forestal mediante sucesión secundaria, aunque aún se sabe poco sobre cómo distintos usos del suelo alteran dicha sucesión (Chazdon y Guariguata 2016). Además de la mencionada mitigación del cambio climático, la regeneración de las selvas logra que el dosel y los ciclos biogeoquímicos sean más cerrados que en los espacios abiertos, contrarrestando la pérdida de nutrientes del suelo y rehabilitándolo para la agricultura (Guariguata y Ostertag 2001). Eso es aprovechado mediante distintas formas de agricultura itinerante, revisadas por Aweto (2013): se talan globos de terreno pequeños ( $\leq 1 \mathrm{ha}$ ), luego se troza y deja que los microorganismos descompongan lo talado; o se quema, cuando el clima lo permite, mineralizando nutrientes útiles para los cultivos de crecimiento rápido (Aweto 2013). Después, se siembran y permiten brotar decenas de especies comestibles, medicinales y maderables. Finalmente, se deja de labrar el terreno después de 2-3 cosechas para que el bosque se regenere. Conforme la regeneración avanza, se extraen selectivamente recursos maderables y no maderables para uso doméstico (Aweto 2013). En cambio, otros agricultores establecen grandes mono- y oligocultivos, ganaderías y otras actividades lucrativas, de modo que los bosques que rebrotan cambian según cómo se usó cada sitio. Así, cada cultivador protagoniza una historia de uso única, tomando decisiones que, luego de abandonar el lote, alteran la sucesión secundaria con respecto a lo que hicieron otros cultivadores (Chazdon 2003, Garrido-Pérez y Glasnović 2014). Tales decisiones obedecen a los contextos natural, socioeconómico y personal (Garrido-Pérez y Glasnović 2014). Según dichos autores, las decisiones de uso del suelo son influenciadas por factores como el clima, el suelo y la distancia a los cuerpos de agua, combinados con fuerzas conductoras socioeconómicas (e.g. presiones de mercado) y políticas (e.g. regulaciones de la tala). Otras fuerzas conductoras son las tecnológicas (e.g. carreteras, instrumentos de labranza), las tradiciones (modos de trabajar la tierra aprendidos de los antepasados), así como asuntos estocástico-individuales (e.g. abandonar un lote para ir a estudiar a la ciudad) (Garrido-Pérez y Glasnović 2014). Si ello es así, y si la regeneración de los bosques varía según el uso que cada propietario le de a su terreno, entonces deben existir asociaciones entre los contextos histórico y socioeconómico que determinan las decisiones de uso del suelo, por un lado, y las características que adquieren los ecosistemas forestales durante la sucesión secundaria, por el otro (ver también Chazdon 2003, Bürgi et al. 2016).

Lo que hacen los humanos sobre un lote se refleja con retardo en los ecosistemas (Chazdon 2003, Bürgi et al.
2016). Garrido-Pérez y Glasnović (2014) respaldan eso argumentando que los árboles son organismos sésiles y más longevos que los humanos por lo que sus individuos, poblaciones y comunidades tardan décadas en crecer y estructurarse después del impacto antrópico, reteniendo señales de impactos ocurridos en el pasado, tales como cicatrices de poda, tocones post-tala, y reclutamiento pulsado debido a remoción, alterándose la estructura del bosque resultante (Garrido-Pérez y Glasnović 2014). Ello explica que propiedades como la densidad (y biomasa aérea) que adquieren los bosques secundarios sean menores en los sitios donde el impacto durante el uso del suelo fue mayor (e.g. Pascarella et al. 2000). Con base en todo eso se propone la hipótesis de trabajo que las características de los bosques secundarios de hoy son el resultado de los distintos disturbios que en el pasado hicieron los humanos allí. Como corolario, la historia y el contexto socioeconómico en torno a los bosques deben permitir la identificación de prácticas de impacto severo, y la propuesta de prácticas que viabilicen la sucesión secundaria.

Este trabajo se realizó con los siguientes objetivos: (a) agregar conocimientos sobre cómo diferentes historias de uso del suelo explican la estructura y la biomasa aérea que, durante la sucesión secundaria, adquieren los bosques amazónicos. (b) Formular sugerencias sobre cómo conservar esos bosques, con base en las historias y contextos socioeconómicos que causaron su transformación.

Se responden las siguientes preguntas:

1. ¿Cuál es el contexto socioeconómico y cuáles fueron las historias del uso que se dio al suelo en lo que hoy son bosques secundarios cercanos, con condiciones abióticas similares?

2. ¿Cuáles son las diferencias en la estructura y biomasa aérea de los árboles del dosel $(\geq 10 \mathrm{~cm}$ de diámetro a la altura del pecho (dap) a $1,3 \mathrm{~m}$ sobre el suelo) entre esos bosques secundarios?

3. ¿Cómo explica la historia dichas diferencias; cuáles prácticas de impacto fueron más severas y cuáles otras viabilizaron mejor la recuperación de la estructura y biomasa aérea del bosque?

4. ¿Qué tendencias de recuperación de la densidad de individuos, el área basal y la biomasa aérea a través de la sucesión secundaria, asociadas a la historia de uso del suelo, bosqueja este estudio, en combinación con otras investigaciones en bosques amazónicos maduros (Pitman et al. 2001, Baker et al. 2004, Pallqui et al. 2014)?

\section{MÉTODOS}

Lugar del estudio. Este estudio se realizó en los alrededores de Atacapi, comunidad de indígenas Kichwa en Muyuna, Cantón Tena, Napo, Ecuador (aprox. 7 km de la ciudad de Tena; 057'11,76” S; 7751'36,49” O), cercana a la Reserva Biológica Colonso-Chalupas (figura 1). Los 
ríos Tena, Pashimbi y Colonso drenan el área. Es zona de bosque húmedo tropical de tierra firme; altitud $\sim 650 \mathrm{~m}$ s.n.m. La temperatura media anual es de $22-24{ }^{\circ} \mathrm{C}$; lluvias de $\sim 4.100 \mathrm{~mm}^{\text {año }}{ }^{-1}$, los suelos son inceptisoles bien drenados (MAGAP 2011).

Evaluación socioeconómica e histórica. Se obtuvieron y consultaron: una imagen de satélite Landsat 7 (ruta 9, fila 60, año 2012) y un mapa catastral de la zona estudiada correspondiente al año 2012 (DHUA 2014). Se efectuaron reconocimientos sobre el terreno para identificar los diferentes bosques secundarios y zonas cultivadas y abiertas para interpretar los usos del suelo en la imagen satelital. Otras imágenes y catastros no pudieron obtenerse, por lo que se usaron métodos etnográficos (Garrido-Pérez y Glasnović 2014), especialmente entrevistas narrativas a 15 adultos (cuadro 1). No se accedió a datos del total de habitantes de Atacapi, pero dicho poblado es parte de

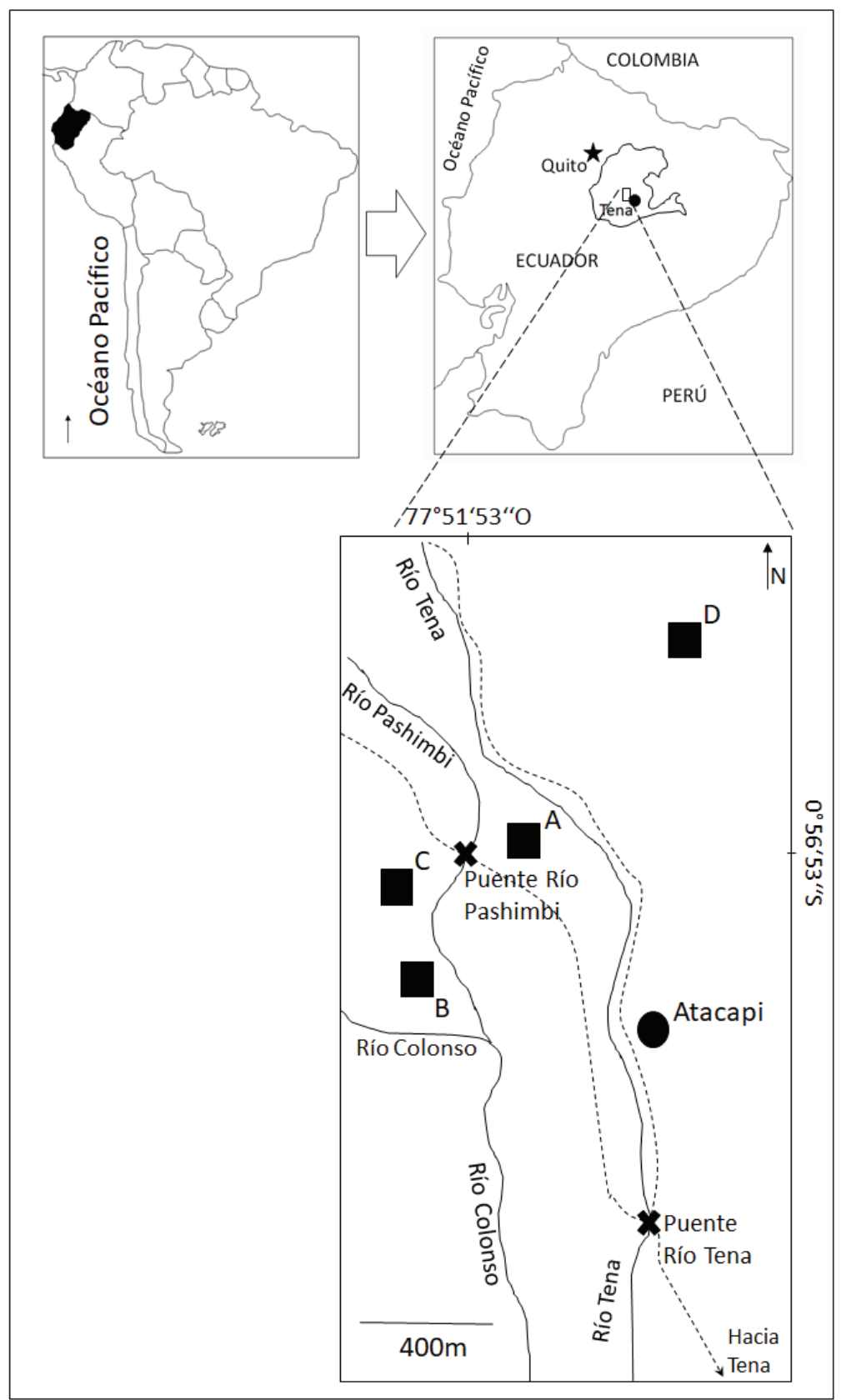

Figura 1. Ubicación de la zona de estudio y de las cuatro parcelas (A, B, C y D) de una hectárea en Atacapi (Napo, Ecuador). Las líneas punteadas en el recuadro inferior representan calles y caminos no pavimentados.

Study site and location of four one-hectare plots (A, B, C and D) in Atacapi, (Napo, Ecuador). Roads are represented as discontinuous lines inside the square on lower panel. 
Cuadro 1. Características de las 15 personas clave entrevistadas para la reconstrucción de la historia de uso del suelo de bosques secundarios amazónicos en Atacapi (Napo, Ecuador). Las entrevistas se hicieron en castellano. $\mathrm{M}=$ masculino, $\mathrm{F}=$ femenino.

Characteristics of 15 key interviewees contacted for the reconstruction of the land-use histories of Amazonian, secondary forests in Atacapi (Napo, Ecuador). Interviews were made in Spanish. $\mathrm{M}=$ male, $\mathrm{F}=$ female.

\begin{tabular}{clcc}
\hline & \multicolumn{1}{c}{ Oficio principal } & Género & Etnia \\
\hline 1 & Agricultor de chakra-ushun-purun; propietario de la parcela D & M & Kichwa \\
2 & Agricultor de chakra-ushun-purun; propietario de la parcela D (hijo del primer entrevistado) & $\mathrm{M}$ & Kichwa \\
3 & $\begin{array}{l}\text { Comerciante y co-propietaria de las parcelas A y B junto a su padre cuando se realizaron los } \\
\text { usos del suelo de este estudio }\end{array}$ & $\mathrm{F}$ & Mestiza \\
4 & Agricultora de chakra-ushun-purun & $\mathrm{F}$ & Kichwa \\
5 & Hijo de la agricultora de chakra-ushun-purun antedicha & $\mathrm{M}$ & Kichwa \\
6 & Agricultora de chakra-ushun-purun & $\mathrm{F}$ & Mestiza \\
7 & Agricultora de chakra-ushun-purun & $\mathrm{F}$ & Mestiza \\
8 & Agricultora de chakra-ushun-purun & $\mathrm{F}$ & Mestiza \\
9 & Agricultora de chakra-ushun-purun & $\mathrm{F}$ & Mestiza \\
10 & Agricultor de chakra-ushun-purun & $\mathrm{M}$ & Kichwa \\
11 & Agricultor de chakra-ushun-purun & $\mathrm{M}$ & Kichwa \\
12 & Funcionario público en Tena & $\mathrm{M}$ & Mestiza \\
13 & Maestro jubilado y operador de un agriturismo cerca Tena & M & Kichwa \\
14 & Agricultor de chakra-ushun-purun; chamán residente en otro poblado & M & Kichwa \\
15 & Agricultor de chakra-ushun-purun, consultor científico residente en otro poblado & M & Kichwa \\
\hline
\end{tabular}

la Parroquia Muyuna que posee 1335 habitantes (GAD Municipal de Tena 2016). Eso, junto a observaciones en eventos públicos de la localidad, sugiere que en Atacapi deben habitar aproximadamente 300 personas, muchas de ellas emparentadas (se observó que tres apellidos son muy comunes). Ello indica que los 15 entrevistados representan aproximadamente un $5 \%$ de la población total. Considerando que los niños componen un amplio conjunto de la población, es muy probable que los 15 entrevistados representen más de un $15 \%$ de los habitantes en capacidad de suministrar información histórico-etnográfica. Es más, el uso del suelo, aunque protagonizado por las amas de casa, es una tarea altamente familiar y estas suelen tener cinco o más miembros. Esto apunta a que los informantes representan un conocimiento del uso del suelo de la región más representativo de lo que sugiere su número.

Casi todos los entrevistados habitan en torno a las parcelas; en un radio de $700 \mathrm{~m}$ con respecto al puente de Pashimbi (figura 1). Dos entrevistados son propietarios de la parcela D y un tercero fue propietario de las parcelas A y $\mathrm{B}$; todos nacieron y crecieron en la zona. Cada persona fue entrevistada al menos tres veces, aunque a quienes fueron propietarios o trabajadores de alguna parcela se les entrevistó hasta seis veces. Dos informantes clave (propietarios de la parcela $\mathrm{D}$ que además habían crecido y trabajado en las fincas correspondientes a las parcelas A, B y C durante varias décadas) fueron contratados para ayudarnos en el trabajo de campo, aprovechando lo cual se les entrevistaba dos-tres veces a la semana por aproximadamente seis meses, conversando seis horas al día. Las entrevistas se hicieron con base en los métodos etnogáficos (e.g., Albuquerque et al. 2010). También se consultaron otros trabajos; sobre todo Vitery (2015).

Las entrevistas se efectuaron entre octubre de 2014 y mayo de 2016. A todos se les explicó que se trataba de una investigación sobre la flora y la historia del lugar indicándoles que no dijeran- ni permitieran que se les preguntase nada que ellos no quisieran responder. También se les dieron los correspondientes términos de consentimiento libre y autorizado. El total de entrevistadores fue de cuatro, pero para cada entrevista los entrevistadores presentes fueron dos. Todos los entrevistadores estaban entrenados y después reportaban a un solo diario escrito. La información se analizó para identificar los actores (que permanecen anónimos en este reporte), factores y fuerzas conductoras de cambio ambiental (Garrido-Pérez y Glasnović 2014), para articular los eventos y causas de disturbio y regeneración de los bosques en líneas de tiempo cuyas fechas se dedujeron de las biografías orales de los entrevistados.

Emplazamiento de las parcelas, mediciones de los árboles y procesamiento de los datos. La parcela A fue ubicada $\mathrm{a} \sim 200 \mathrm{~m}$ del río Tena y otros $\sim 200 \mathrm{~m}$ del río Pashimbi. La parcela B está a $\sim 100 \mathrm{~m}$ del río Pashimbi y $\sim 200 \mathrm{~m}$ 
del río Colonso. La parcela $\mathrm{C}$ se ubicó a $\sim 200 \mathrm{~m}$ del río Pashimbi y la parcela D a $\sim 600 \mathrm{~m}$ del río Tena. Las condiciones climáticas, así como comparaciones preliminares entre suelos (Garrido-Pérez et al. 2017) indican que estos factores no varían entre los parches estudiados. Cada parcela midiólha $(50 \mathrm{~m} \times 200 \mathrm{~m})$ y fue trazada siguiendo el contorno de su terreno. Todas las plantas leñosas con dap $\geq 10 \mathrm{~cm}$ fueron etiquetadas y medidas en cada parcela (método usado por la Red Amazónica de Inventarios Forestales -RAINFOR para bosques no intervenidos) (e.g., Baker et al. 2004, Pallqui et al. 2014). Los indicadores de estructura de las selvas fueron: densidad de individuos, área basal $\left(\mathrm{m}^{2} \mathrm{~h}^{-1}\right)$ y área basal promedio $\left(\mathrm{m}^{2}\right.$ árbol $\left.{ }^{-1}\right)$. Se hicieron histogramas con la distribución de diámetros arbóreos para detectar diferencias de estos entre parcelas (prueba de Kruskal-Wallis). Se calculó la biomasa aérea de los árboles usando la siguiente fórmula de Cuenca et al. (2014): $\mathrm{BT}=-26,63+0,42 \mathrm{dap}^{2}$. Se eligió dicha fórmula porque se basa en los diámetros arbóreos (no se midieron longitudes) y porque, de las dos fórmulas basadas en diámetros reportadas por los autores antedichos, es la que se basaba en un mayor número de especímenes ( $\mathrm{n}=22$ árboles; coeficiente de determinación $\mathrm{R}^{2}=0,86$ ). Luego de calcular la biomasa aérea de cada árbol, se transformaron los resultados a toneladas por hectárea. Finalmente, se compararon gráficamente los datos con los de los bosques amazónicos maduros de tierra firme reportados por Pitman et al. (2001), Baker et al. (2004), Pallqui et al. (2014) para ilustrar cambios en la sucesión secundaria asociados a la historia de uso del suelo.

\section{RESULTADOS}

Evaluación del contexto socioeconómico. Las actividades económicas de los moradores Kichwa en 2014-2016 incluyeron: (1) agricultura itinerante de roza-tumba-trozaquema. (2) Extracción de recursos forestales. (3) Trabajo eventual asalariado, -mayormente los hombres; sobre todo la albañilería en la ciudad de Tena. (4) Trabajo semi-permanente en comercios y restaurantes de Tena y la vecina Archidona. (5) Guía eventual a turistas y científicos. (6) Labores para una universidad fundada en octubre de 2014 y construida con materiales provisionales de fuera de la zona sobre un potrero abandonado y pantanoso rellenado con tierra. Los jardines domésticos de los Kichwa incluyen algunas gallinas, plantas medicinales y ornamentales. Ninguna familia tuvo cerdos (Sus scrofa L.) ni vacas (Bos taurus L.); solamente un hogar tuvo un caballo (Equus ferus L.). El principal medio de transporte para distancias $\sim 5 \mathrm{~km}$ es la bicicleta. Para distancias mayores se usan motocicleta y -mayormente, autobuses provenientes desde Tena; no fueron vistos más de tres carros en las residencias. Todo esto implica un nivel de integración a la economía de mercado relativamente bajo y una escasa capacidad de movilizar productos hacia y desde los hogares, campos y bosques.
Una fuente independiente de este estudio (Vitery 2015) confirmó ante Naciones Unidas algo que explicaron varios informantes de esta investigación: la agricultura itinerante de los Kichwa es protagonizada por las mujeres, aunque con participación de los demás familiares. Vitery (2015) indicó que el proceso consta de las siguientes tres fases: (a) Chakra: se clarea un terreno por roza-tumba-troza y quema luego de decidir en familia qué terreno será. Se hacen viveros con semillas de especies medicinales, frutales y maderables recolectadas de los alrededores. Se plantan especies de ciclo corto; especialmente yuca (Manihot esculenta Crantz, Euphorbiaceae). (b) Ushun: cumplida la primera cosecha de yuca (noveno mes) se siembran los plantones del semillero, haciéndolos co-existir con las especies de ciclo corto. (c) Purun: a partir del sexto año, el lote consta de un barbecho natural que co-existe con los árboles plantados. El manejo sigue conforme avanza la sucesión. Allí los hombres les transmiten a sus hijos de manera demostrativa sus conocimientos sobre las plantas (Vitery 2015).

Un lote trabajado así puede llegar a tener 62 especies manejadas, de las cuales 44 son árboles (Vitery 2015). Son cifras similares a las suministradas por dos de los informantes y la observación de fincas de este estudio ( $>55$ especies en media hectárea). Las especies más comúnmente observadas, sobre todo en el período de chakra, fueron: yuca (M. esculenta; cultivo principal), maíz (Zea mays L., Poaceae), frijoles (Phaseolus spp., Fabaceae), bananos y plátanos (Musa spp., Musaceae). El periodo de ushun incluye -además de yuca, cítricos (Citrus spp., Rutaceae), chonta o pixvae (Bactris gasipaes Kunth, Arecaceae), morete (Mauritia flexuosa L.f., Arecaceae), coco (Cocos nucifera L., Arecaceae) y otras palmas, cacao blanco (Theobroma bicolor Humb. Et Bonpl., Sterculiaceae), guabas (Inga spp., Fabaceae) y aguacate (Persea americana Mill., Lauraceae). También estimulantes ricos en antioxidantes como: guayusa (Ilex guayusa Loes., Aquifoliaceae), cacao (Theobroma cacao L., Sterculiaceae) y café robusta (Coffea canephora Pierre ex A. Froehner, Rubiaceae); así como condimentos como el ají (Capsicum spp., Solanaceae). Durante el purun, muchas de las especies plantadas durante el ushun coexisten con las especies silvestres que brotan durante la sucesión.

Por su parte, la minoría de pobladores criollos y mestizos: (1) (semi)-monocultiva comercialmente especies como T. cacao, Citrus spp., C. canephora L., e I. guayu$s a$. (2) Cría cerdos, caballos (arrieros y "finos"), y vacas. (3) Posee o gerencia restaurantes y hosterías para turistas y estudiantes. (4) Hay una finca con $>250$ ha de espacios abiertos, entre los cuales se incluyen estanques para la cría de peces (tilapias, Oreochromis sp.). (5) Otros tienen casas en la zona y la frecuentan, pero alternan eso con sus vidas en Tena y otras ciudades, por lo que su arribo, asociado al asfaltado de una carretera en 2011 , está urbanizando el área. Varios usan automóviles sedán, carros de doble tracción y "pick-ups" con capacidad de carga de $\sim 2 \mathrm{Mg}$; no 
hubo camiones. Estos datos sugieren que, en comparación con los kichwa, están más integrados al mercado, aunque sus ventas y producción siguen siendo bajas.

La universidad es estatal y ocupa una finca de aproximadamente 260 ha. Fue fundada en octubre de 2014 y tiene $\sim 500$ estudiantes más $\sim 100$ docentes y empleados. La rodean 25 fincas que cubren 741,9 ha; un $98 \%$ de esa superficie lo usan los indígenas (DHUA 2014). El Estado también está presente mediante del Ministerio del Ambiente del Ecuador (MAE). Este administra la Reserva de la Flora y Fauna Colonso-Chalupas, cuya entrada está a menos de 3,5 km al oeste del puente sobre el río Pashimbi. La cacería, la tala y la extracción de recursos de la reserva están prohibidas. La extracción y venta de madera, incluso de cada finca, están estrictamente reglamentadas, aunque las leyes cambian en períodos tan cortos como cinco años. Empero, los diámetros mínimos de corta generalmente son de $50 \mathrm{~cm}$. Otras influencias externas provienen del mercado de I. guayusa para consumo por los restaurantes y habitantes de Tena (ciudad de 60.880 habitantes) (GAD Municipal de Tena 2016). Una empresa extranjera exporta hojas de dicha planta. No hay explotaciones petroleras ni aserraderos en el lugar estudiado, pero en zonas aledañas hay 10 aserraderos pequeños y poco tecnificados que convierten rollos de Piptocoma discolor Kunth (Asteraceae) en cajas para legumbres (Erazo et al. 2014).

Vegetación circundante. La parcela A estuvo en un parche de bosque secundario menor que 2 ha rodeado de: (a) pasturas abandonadas aproximadamente en 2013. (b) Cítricos ( $\sim 0,5$ ha). (c) Dos casas, una de ellas incompleta. (d) La carretera. La parcela B estuvo rodeada de un bosque secundario con al menos dos zonas: una "joven" donde se colocó la parcela B y otra más madura, donde fue emplazada la parcela $\mathrm{C}$ (ver edades sucesionales en los resultados). A partir de $\sim 850 \mathrm{~m}$ al oeste de parcela B el bosque secundario da lugar a otro más viejo, aunque con dosel irregular. Esto sugiere una vigorosa formación de claros por caída (y/o derribo) de árboles. No fueron vistos indicios de tala; sí de árboles tumbados por el viento. También, el lado del río Pahismbi opuesto a donde está la parcela B (figura 1) lo ocupa el espacio abierto de lo que, hasta 2013, era potrero. La parcela $\mathrm{C}$ estuvo rodeada por el mismo bosque que la parcela $\mathrm{B}$, aunque en una zona donde aquel es más viejo, sobre una meseta a $\sim 4 \mathrm{~m}$ por encima de la parcela B. La meseta forma un risco a cuyo fondo está el río Pashimbi ( $\sim 8 \mathrm{~m}$ hacia abajo). Las parcelas $\mathrm{B}$ y $\mathrm{C}$ tienen en su interior pendientes suaves que llevan a un arroyo.

La parcela D se ubicó a $\sim 550 \mathrm{~m}$ del río Tena (figura 1), en terrenos de una familia Kichwa, sobre una meseta a aprox. $10 \mathrm{~m}$ de altura con respecto al río Tena. La parcela incluye un arroyo grande, aunque este se ubica luego de un risco de $90^{\circ}$ con respecto a la horizontal. El bosque donde se estableció la parcela $\mathrm{D}$, además de la finca de los dueños, abarca terrenos de otras fincas de indígenas Kichwa. Ello rodeó a la parcela D y su rodal de plantíos Kichwa, vi- viendas con traspatios, y bosques secundarios de distintas edades. Nada de eso circundaba a las otras tres parcelas.

Evaluación de historias de uso del suelo. Todas las edades sucesionales que se indican a continuación son con respecto al año 2015 (figura 2). El bosque de la parcela A tiene una edad de 12-15 años, en regeneración desde 2003. Durante su crecimiento, el $50 \%$ del área del bosque fue dehesado y desmalezado. Fueron vistos tocones de brinzales que indican que dicha práctica continúa. Una red de caminos sugiere que también fue y sigue siendo transitada intensamente a pie. Después de 1980, el terreno fue fumigado una vez con herbicidas no identificados. Entre 1980 y 2003, una zona equivalente a la mitad de la parcela A era parte de un potrero (número de reses desconocido), pero menor al que pastaba allí durante los años 1970 (figura 2). Entre 1980-2003, la otra mitad de la parcela estaba separada del ganado: tenía un oligocultivo de plantas perennes de los que fueron vistos algunos árboles de C. canephora y cítricos. Entre los años 1980 y 2003 la matriz circundante incluía un espacio semi-abierto con canelos (Nectandra y Ocotea spp, Lauraceae) y guayacanes (Tabebuia guayacan Hemsl., Bignoniaceae), además del potrero.

El bosque de la parcela B tiene 22-25 años (en regeneración desde $\sim 1991$, figura 2). Perduran fuera de la parcela corredores de aprox. seis metros de ancho por donde pasaban vehículos. Se hallaron restos de una cerca: alambres de púas y estacas de madera curada y cortada en tablas. La trayectoria de las estacas atravesaba diagonalmente la parcela, separando una sección de $\sim 40 \%$ del área de otra de $\sim 60 \%$ de la hectárea. Aquel $40 \%$ parece haber estado dedicado mayormente a la ganadería y ofrecía al ganado la oportunidad de beber de un arroyo que hay en la parcela, y tal vez de las inmediaciones del río Pashimbi.

En una meseta de la parcela B se hallaron: un alero de zinc, fragmentos de parales, de madera curada y labrada para paredes, una pequeña acera de aprox. $2,5 \mathrm{~m} \times 0,5 \mathrm{~m}$, fragmentos de un piso de cemento, y restos de un rollo de alambre de púas. Eso correspondió a una vivienda de $28 \mathrm{~m}^{2}$ según mediciones de campo, aunque estas se basaron en las posiciones del fragmento del piso y los pedazos de los parales. Los informantes indicaron que la habitó una persona solitaria, no indígena, quien falleció y, luego, el terreno quedó abandonado. Los materiales señalados sugieren que el habitante podía adquirir insumos de afuera. Se criaba ganado menor como guatuzas (Dasyprocta spp.) y cerdos. El uso de leña para cocinar puede considerarse como poco intenso debido al bajo número de habitantes. De acuerdo con los informantes, y con observaciones en otras fincas de mestizos, en torno a la casa había algunos cultivos perennes como: plátanos (Musa spp.), yuca (M.esculenta), guabas (Inga spp). De estas últimas quedaron algunos individuos como legado. En el espacio mayor de la parcela ( $\sim 60 \%$ de la hectárea) se cultivaron naranjas (Citrus sinensis Pers.), toronjas (Citrus x paradisi Macfad.) y limones (Citrus limon L.; todos Rutaceae). Esto de acuerdo 


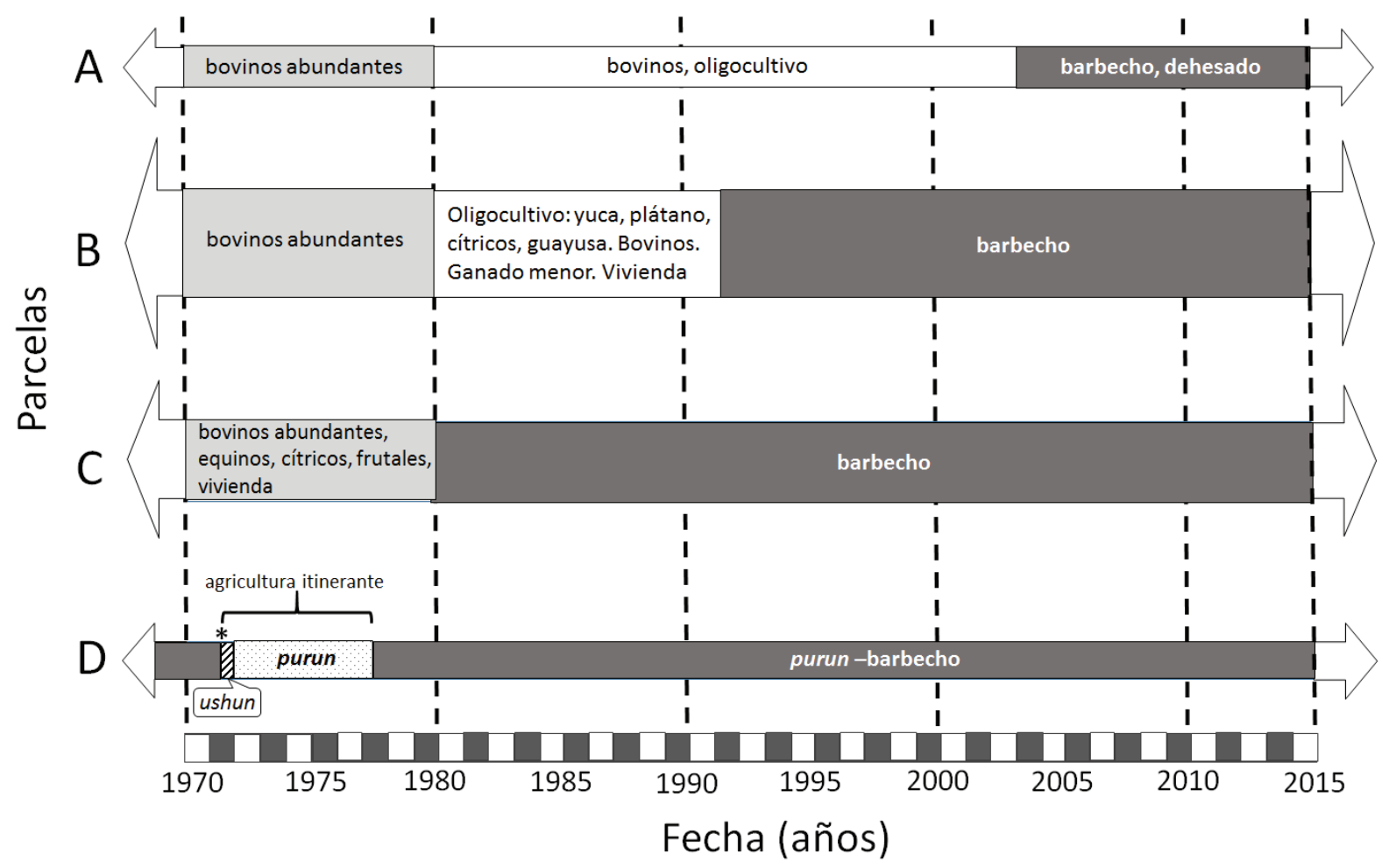

Figura 2. Historias de uso del suelo de cuatro parcelas de una hectárea en bosques secundarios amazónicos de tierra firme en Atacapi, Napo, Ecuador. El asterisco indica el momento en que el lote que ocupa la parcela D se clareó para la siembra.

Land-use histories of four one-hectare plots in secondary, Terra Firme Amazonian forests in Atacapi, Napo, Ecuador. The asterisk (*) shows the moment where the stand currently occupied by plot D was clear-cut before planting.

con los informantes, y con algunos de esos árboles que aún quedaron. La producción de cítricos requirió de desmalezado periódico, lo cual implicó el frecuente caminado de personas con machete. Todo esto sucedió entre aprox. 1980 y 1991 (figura 2). Previo a eso (década de 1970s), el lote estaba englobado en la misma matriz de ganadería abundante en que estaba la parcela $\mathrm{A}$.

El bosque en la parcela $C$ tiene $\sim 35-37$ años (en regeneración desde 1980 , figura 2). Entre 1970-1980 el lote tuvo una edificación de madera con patio, una caballeriza (aproximadamente ocho caballos; ahora ocupada por abundantes Selaginella sp.), parte de un potrero ( $\sim 300$ reses), y árboles frutales. No hubieron rastros de la infraestructura ni de cercas o pilotes; solo una fosa (presunta letrina) ahora tapada con tierra y plantas. Entre la parcela y el río hay un camino (todavía usado pese al cierre del dosel), por donde propietarios, arrieros, empleados y visitantes solían entrar y salir tanto a pie como a caballo. Hacia el extremo opuesto a la edificación, la parcela tuvo cítricos y otros árboles frutales. Este período de uso (1970-1980) coincide con el de ganadería abundante en las parcelas A y B.

El bosque de la parcela D tiene 35-40 años (en regeneración desde aprox. 1977) pero con un manejo de purun entre 1972-1977 y lustros posteriores (figura 2). Aunque la familia tuvo $\sim 30$ reses, el lote que ocupa la parcela fue usa- do solo para agricultura itinerante por la matriarca. Consecuentemente, tuvo un periodo de ushun (aprox. 1972) después del clareo (chakra, figura 2). El número de especies e individuos plantados se desconoce. Algunas especies cultivadas fueron: M. esculenta, Z. mays, Musa spp., B. gasipaes, T. bicolor, T. cacao, guabas "pakai" (Inga edulis Mart., Fabaceae), P. americana, algunos cítricos y maderables. Por tratarse de un sistema agroforestal multiestratificado, el desmalezado no fue homogéneo ni regular o intenso. Por ejemplo, durante el ushun el desmalezado era menos intenso y frecuente que en los oligo-cultivos. La poda prácticamente no se practicaba. Conforme la selva se regeneraba (periodo purun), las especies plantadas inicialmente co-existían con las plantas perennes. Pero al caminar a veces se cortaban brinzales con el machete, por ejemplo, en torno a los de especies eventualmente útiles como madera. Esta se extrajo esporádica y selectivamente según necesidades como el reemplazo de vigas o parales en la casa, la cual estaba fuera del lote donde se estudiaron los árboles. Tales manejos persistieron durante la sucesión secundaria ( 1977-presente).

Estructuras y biomasa aérea. Las cuatro parcelas juntas tuvieron 1.559 árboles. Parcelas $\mathrm{A}=323, \mathrm{~B}=332, \mathrm{C}=489$ y $\mathrm{D}=415$ individuos. Los diámetros de los árboles varia- 
ron mucho entre parcelas (errores estándar, cuadro 2) mayormente por los árboles grandes en algunas parcelas. Ello sugiere que -al clarear, los usuarios del suelo dejaron árboles remanentes que dieron sombra. Los diámetros de los árboles en las parcelas B (mediana $=16,7 \mathrm{~cm} ; \mathrm{n}=332$ ) y C (mediana $=17,1 ; \mathrm{n}=489)$ fueron similares, pero menores a los de las parcelas A (mediana $=19,9 \mathrm{~cm} ; \mathrm{n}=323$ ) y D (mediana $=19,4 \mathrm{~cm} ; \mathrm{n}=415)$ cuyos valores fueron semejantes entre sí (Kruskal-Wallis, $P<0,001$; ver medias en el cuadro 2); pese a que el rodal en $\mathrm{D}$ era mucho más viejo que en A (cuadro 2). Puesto que la prueba de KW también compara distribuciones, en la variación de los diámetros en las parcelas A y D también predominaron los árboles delgados en comparación con las otras dos parcelas. Ello sugiere que el manejo durante la sucesión secundaria; es decir, durante el periodo de purun, "rejuveneció" al bosque en la parcela D (bosque de 35-40 años), haciendo que sus clases diamétricas se asemejaran a un barbecho de tan solo 12-15 años (parcela A) y no a las de su bosque contemporáneo (parcela C; figura3). En los histogramas, fueron comparativamente profundos: el segundo valle en el de la figura 3B-D, y el tercer valle en la figura 3C. Ello, sugiere que, durante la sucesión secundaria, pudo haber derribo de árboles por el viento y tala selectiva en más de una parcela.

El área basal promedio varió poco entre parcelas (cuadro 2) lo cual sugiere que hubo un efecto pobre de los árboles más grandes en la estructura del bosque en unas parcelas con respecto a las otras, y que la sucesión secundaria homogenizó con rapidez al área basal promedio a pesar de las distintas historias de uso del suelo. En contraste, las variaciones de las áreas basales totales entre parcelas se tradujeron en cambios amplios de la biomasa aérea (última columna del cuadro 2) en el siguiente orden: $\mathrm{C}>\mathrm{B}>\mathrm{D}>\mathrm{A}$. Dicho orden no es plenamente consistente con el de las
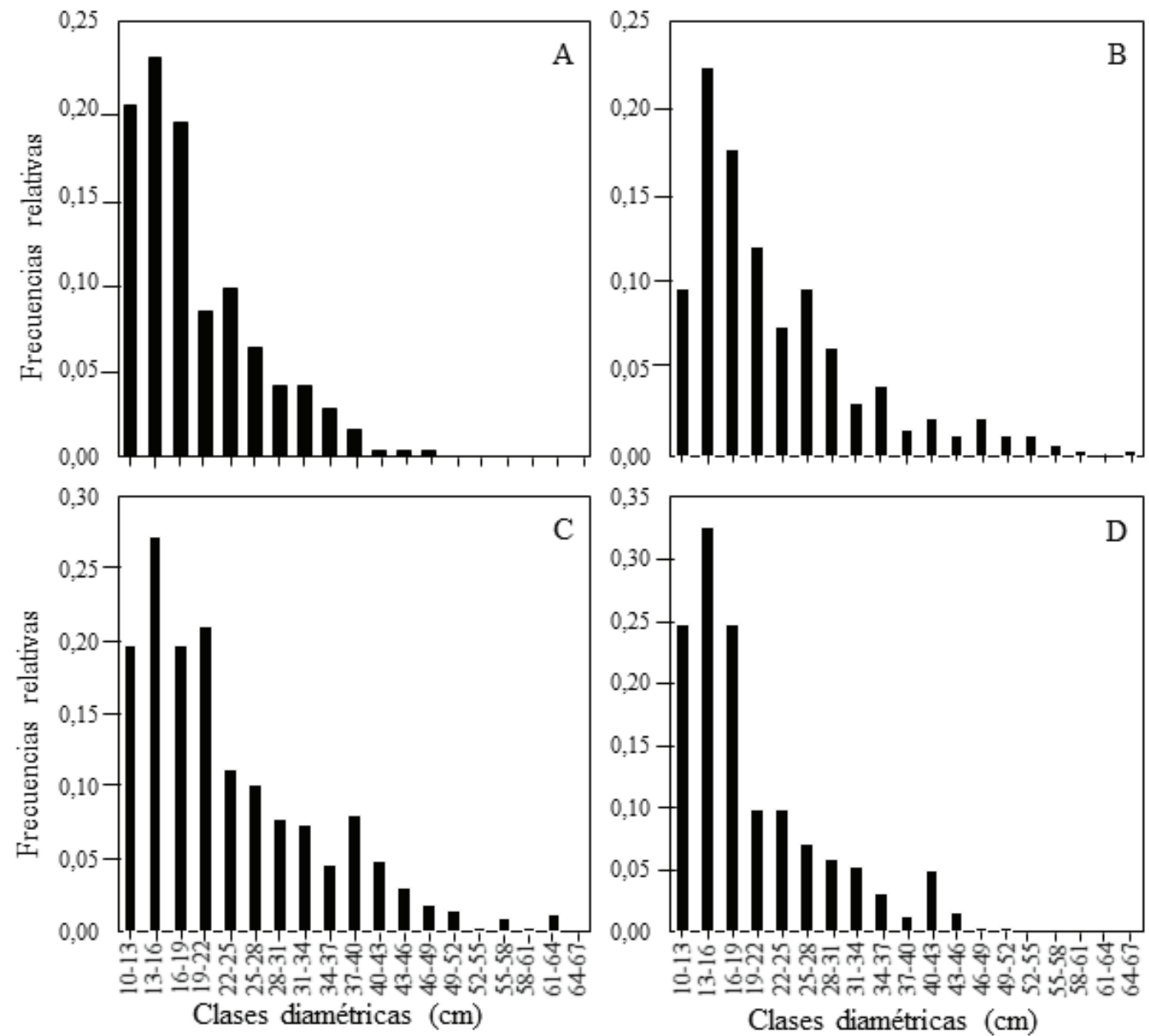

Figura 3. Distribuciones de frecuencias de las clases diamétricas $(\mathrm{cm})$ de los árboles de diámetro a la altura del pecho $(1,3 \mathrm{~m}$ sobre el suelo) $\geq 10 \mathrm{~cm}$ en cuatro parcelas (A, B, C y D) de 1 ha en bosques secundarios en la amazonia ecuatoriana. Prueba de Kruskal-Wallis, $P<0,001$.

Frequency distribution of diameter classes of trees $\geq 10 \mathrm{~cm}$ diameter at breast height (1.3 $\mathrm{m}$ above ground) for four one-hectare plots (A, B, $\mathrm{C}$ and D) in secondary forests of the Ecuadorian Amazon. Kruskal-Wallis, $P<0.001$. 
Cuadro 2. Estructuras y biomasas aéreas de las comunidades de árboles $\geq 10 \mathrm{~cm}$ de diámetro a la altura del pecho $(1,3 \mathrm{~m}$ sobre el suelo) en cuatro parcelas de 1 ha de bosques secundarios con historias de uso del suelo diferentes en la amazonia ecuatoriana. $\mathrm{ABt}=$ área basal total, $\mathrm{ABp}$ = área basal promedio, $\mathrm{BT}=$ biomasa aérea total calculada para cada árbol usando la fórmula de Cuenca et al. (2014) $\mathrm{BT}=-26,63+0,42 \mathrm{dap}^{2}$. Ver las historias de uso en la figura 2.

Structures and aboveground biomass for communities of trees $\geq 10 \mathrm{~cm}$ diameter at breast height $(1.3 \mathrm{~m}$ above ground $)$ for four one-hectare plots with different land-use histories in the Ecuadorian Amazon. $\mathrm{ABt}=$ total basal area, $\mathrm{ABp}=$ mean basal area, $\mathrm{BT}=$ above ground biomass calculated using the formula $\mathrm{BT}=-26.63+0.42 \mathrm{dap}^{2}$ (by Cuenca et al. 2014). See land-use histories in figure 2.

\begin{tabular}{ccccccc}
\hline $\begin{array}{c}\text { Parcela } \\
\text { (edad en años) }\end{array}$ & $\begin{array}{c}\text { Densidad } \\
\left(\text { individuos ha }{ }^{-1}\right)\end{array}$ & $\begin{array}{c}\text { dap } \\
(\mathrm{cm})( \pm \mathrm{EE})\end{array}$ & $\begin{array}{c}\text { dap del árbol } \\
\text { más grande }(\mathrm{cm})\end{array}$ & $\begin{array}{c}\text { ABt. } \\
\left(\mathrm{m}^{2} \mathrm{ha}^{-1}\right)\end{array}$ & $\begin{array}{c}\text { ABp. } \\
\left(\mathrm{m}^{2} \text { árbol }^{-1}\right)\end{array}$ & $\begin{array}{c}\text { BT } \\
\left(\mathrm{Mg} \mathrm{ha}^{-1}\right)\end{array}$ \\
\hline A (12-15) & 323 & $19,47(0,61)$ & 163,06 & 12,60 & 0,04 & 58,79 \\
B (22-25) & 332 & $22,89(3,53)$ & 66,88 & 16,20 & 0,05 & 79,97 \\
C (35-37) & 489 & $23,50(3,30)$ & 62,90 & 25,89 & 0,05 & 125,47 \\
D (35-40) & 415 & $19,41(0,39)$ & 50,96 & 14,17 & 0,03 & 64,78 \\
\hline
\end{tabular}

edades de los rodales $(\mathrm{D} \approx \mathrm{C}>\mathrm{B}>\mathrm{A}$; cuadro 2$)$. Esto sugiere que las edades sucesionales no bastaron para explicar los cambios en la captura de $\mathrm{CO}_{2}$ atmosférico: el bosque de la parcela $\mathrm{D}$, cuyo historial es de purun y barbecho manejado y rodeado de una compleja matriz de chakras, puruns y bosques secundarios manejados, capturó menos $\mathrm{CO}_{2}$ que su contemporáneo de la parcela $\mathrm{C}$, la cual fue una edificación-potrero-oligocultivo, hoy rodeada de bosques secundarios menos manejados.

Comparaciones con otros estudios. La densidad de individuos tendió a aumentar según la edad de los rodales a pesar del bajo número de parcelas estudiadas; lo cual se nota incluso si el observador excluye la parcela $\mathrm{D}$ al mirar la figura $4 \mathrm{~A}$. Ello sugiere que la acumulación de individuos durante la sucesión secundaria progresó en las parcelas independientemente de sus historias de uso del suelo. En contraste, observando la figura $4 \mathrm{~b}$ se nota que la acumulación del área basal sí varió en asocio a la historia de uso del suelo. Cuando se excluye de la observación a la parcela $\mathrm{D}$ al mirar la figura $4 \mathrm{~B}$, los resultados sugieren que los rodales alcanzaron un área basal comparable a la de los bosques maduros después de los 35-37 años. En cambio, al incluir al punto correspondiente a la parcela $\mathrm{D}$ en la observación, se infiere que la recuperación del área basal durante la sucesión se retardó debido al uso del suelo por chakra-ushun-purun y barbecho manejado. También la biomasa aérea tendió a recuperarse con la edad sucesional siguiendo un patrón mucho más predecible cuando se excluye de la observación de la figura $4 \mathrm{C}$ a la parcela $\mathrm{D}$, que cuando se la incluye.

\section{DISCUSIÓN}

$\mathrm{Al}$ igual que otros trabajos (e.g., Pascarella et al. 2000), esta investigación ha usado las ciencias sociales como auxiliares para interpretar los resultados de las mediciones de los árboles, explicando por qué algunos bosques secunda- rios siguen rutas sucesionales distintas a las de otros (ver también Bürgi et al. 2016). Así, para los bosques secundarios estudiados, la tala y el retiro selectivos de árboles durante la sucesión, y su concomitante formación de claros, explican por qué un bosque cercano a los 40 años posee un área basal y una biomasa aérea semejantes a las de otro bosque de apenas $\sim 12$ años, pero con una densidad mayor a la de este último. Contrástese eso con las siguientes explicaciones menos plausibles o parsimoniosas. (a): Es conocido que los cambios espaciales del clima y el suelo alteran las características de los bosques (e.g., EsquivelMuelbert et al. 2017), pero el clima fue semejante entre las parcelas estudiadas, y las observaciones preliminares del suelo indican que este también lo fue (Garrido-Pérez et al. 2017). (b) Procesos densodependientes, como la supresión del crecimiento de un alto número de individuos mediante la competencia por otros en el pasado, son más difíciles de someter a prueba (Connell 1990). Eso en comparación con la historia de uso del suelo.

Las historias de uso del suelo también ayudan a aclarar contradicciones entre estudios de estructura y funciones forestales. En Puerto Rico y República Dominicana se descubrió que la densidad y el área basal son menores en los ex-potreros que en los ex-sistemas agroforestales (Pascarella et al. 2000). Esto pareciera inconsistente con los resultados reportados para la parcela D. Pero, a la luz de la historia, es plausible que lo antedicho se deba a que la parcela $\mathrm{D}$ no fue abandonada, sino que continuó usándose recurrentemente para extracción y tala selectiva durante la sucesión. Según otro estudio (Garrido-Pérez et al. 2017) la misma parcela tuvo mayor diversidad de especies que las otras; algo consistente con la tradición indígena de mantener bosques con una amplia variedad de especies para usarlas (Vitery 2015).

Comparando con los estudios de RAINFOR, los resultados de esta investigación: (a) confirman que las velocidades de recuperación de las características de los bosques 

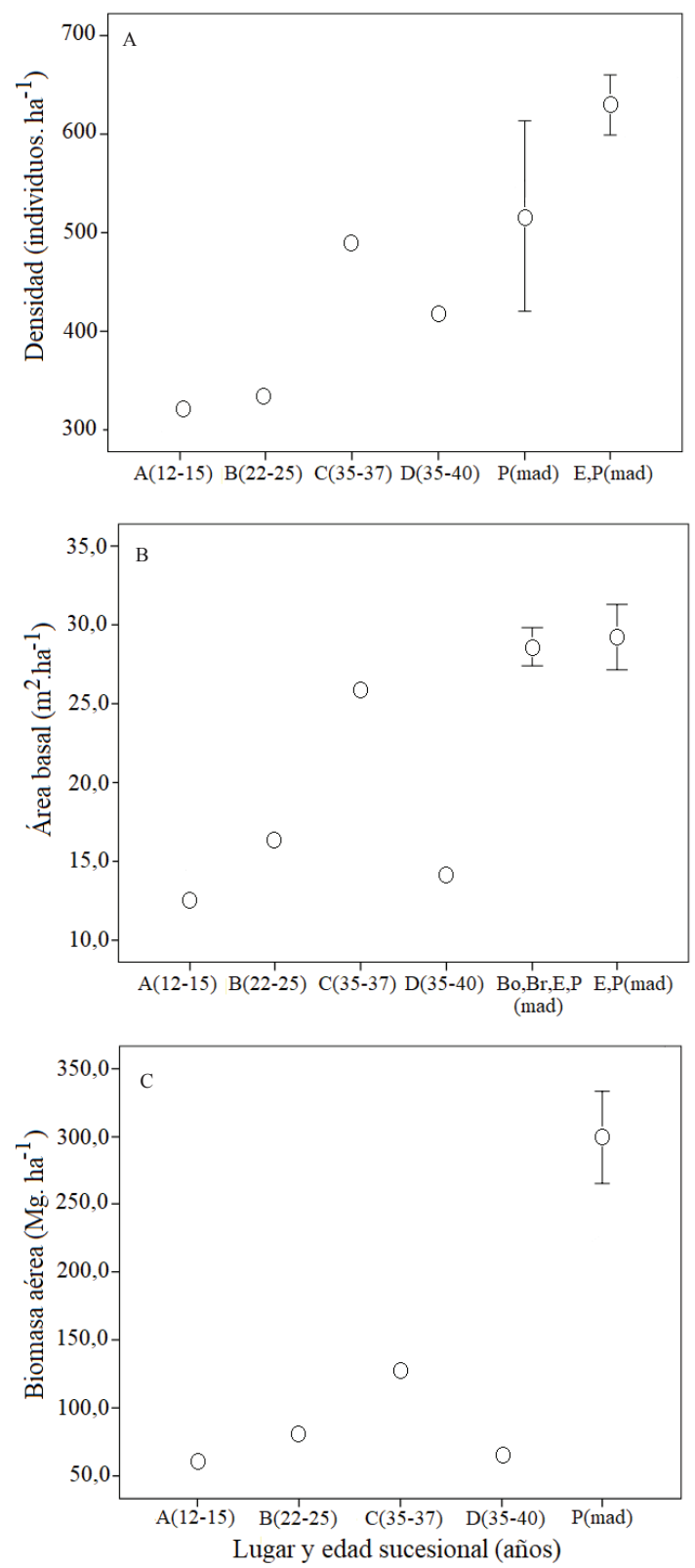

Figura 4. Aumento de la densidad (A), área basal (B) y biomasa aérea $(\mathrm{C})$ de los árboles $\geq 10 \mathrm{~cm}$ de diámetro a la altura del pecho (1,3 m sobre el suelo) en parcelas de 1 ha, según la edad de bosques amazónicos de tierra firme. A, B, C y D son bosques secundarios (este estudio); "mad" significa "bosques maduros". $\mathrm{P}(\mathrm{mad})=$ datos de Tambopata-Madre de Dios, Perú (Pallqui et al. 2014; $\mathrm{n}=9$ parcelas). E,P(mad) = datos de Yasuní y Manu (Ecuador y Perú respectivamente) (Pitman et al. 2001; $\mathrm{n}=26$ parcelas $)$. Bo, Br, E, $\mathrm{P}(\mathrm{mad})=$ datos de Bolivia, Brasil, Ecuador y Perú (Baker et al. 2004; $\mathrm{n}=44$ parcelas).

Increase of tree-density (A), basal area (B), and aboveground biomass (C) for all trees $\geq 10 \mathrm{~cm}$ diameter at breast height (1.3 m above ground) for one-hectare plots according to forest age for Amazonian Terra Firme forests. A, B, C and D are secondary forests (this study); "mad" means "mature forest". $\mathrm{P}(\mathrm{mad})=$ data from Tambopata-Madre de Dios, Peru (Pallqui et al. 2014; $\mathrm{n}=9$ plots). E,P(mad) = data from Yasuni and Manu (Ecuador and Peru, respectively) (Pitman et al. 2001; $\mathrm{n}=26$ plots). Bo,Br,E,P(mad) = data from Bolivia, Ecuador and Peru (Baker et al. 2004; $\mathrm{n}=44$ plots). son alteradas por los disturbios durante el uso (Chazdon y Guariguata 2016). (b) Sugieren que la extracción durante la sucesión retarda la recuperación del área basal y la biomasa (compárese con Pitman et al. 2001, Baker et al. 2004 y Pallqui et al. 2014). Pero (c): la densidad puede recuperarse con cierta rapidez gracias a la colonización desde la matriz circundante y la formación de claros (comparando con Pitman et al. 2001 y Baker et al. 2004). Todo eso puede ayudar a explicar por qué hay ecosistemas forestales tropicales cuyo recobro tarda 50-80 años (Martin et al. 2014), en lugar de aprox. 25 años (Guariguata y Ostertag 2001): los disturbios recurrentes por el uso probablemente retardaron la acumulación de área basal y biomasa detectados por Martin et al. (2014).

Para decidir cómo manejar los territorios, conviene determinar qué fuerzas conductoras (sensu Garrido-Pérez y Glasnović 2014) promueven- o revierten la deforestación. A la luz de la historia ecuatoriana reciente, la reconstrucción histórica aquí efectuada indica que el que tres de las cuatro parcelas amazónicas estudiadas hayan sido ocupadas por ganaderías y oligo-cultivos durante la década de los 1970s, se explica por el aumento en el consumo de alimentos que generaron las exploraciones y explotaciones petroleras en la región a partir de $\sim 1967$ (Rivadeneira 2004). Dicho autor indica que hacia 1980 la explotación se mantuvo, pero las exploraciones bajaron -reduciéndose el número de consumidores con respecto al periodo previo. Se infiere que no todos los usuarios del suelo reaccionaron igual: los de las parcelas A y B redujeron sus hatos y fortalecieron la diversificación; los de la parcela $\mathrm{C}$ no la trabajaron más; los de la parcela $\mathrm{D}$ continuaron con su práctica, autárquica y agro-bio-diversa, de chakra-ushun-purun. También se infiere que, en su calidad de fuerza conductora, el Estado influyó menos que el mercado alimentario en los cambios ambientales: las limitaciones a la tala de parte del MAE contribuyeron más a mantener los bosques que a clarearlos. El propio Estado ha padecido irregularidades: Ecuador ha tenido casi dos Presidentes por cuatrienio entre 1970 y 2010. Así que, con base en las evidencias acopiadas, se interpreta que las fuerzas conductoras que coadyuvaron al cese del uso de las parcelas B y C en 1991 y 1980, respectivamente, fueron: el fallecimiento de los usuarios del suelo y el cambio de actividad económica de sus descendientes ante una menor demanda alimentaria.

El caso aquí estudiado ilustra cómo el aumento de la demanda alimentaria en zonas de frontera agrícola acicatea allí los (cuasi) monocultivos y la ganadería. Cuando se reduce aquella demanda, el uso del suelo cambia: se abandonan algunos lotes, y los bosques que rebrotan allí siguen rutas sucesionales distintas a las que resultan de la agricultura itinerante (Chazdon 2003). Ello porque los agricultores itinerantes amazónicos perciben al bosque como una fuente de valores de uso complementaria a los cultivos, por lo cual los barbechos siguen siendo manejados y utilizados para extraer alimentos, materiales de reparación casera, medicinas y otros valores de uso (Vitery 2015). 
La evaluación aquí publicada sugiere que la demanda de alimentos y viviendas en la zona estudiada puede aumentar futuramente debido a dos fuerzas conductoras que aparecieron en 2014. (1) La universidad fundada en dicho año, la cual atrae estudiantes y personal de otras provincias en número creciente. Ello puede fomentar la urbanización y los (cuasi)monocultivos; si no en Atacapi, al menos en la periferia -recuérdese que, pese a estar a cientos de kilómetros de Atacapi, las exploraciones petroleras provocaron cambios allí. (2) La empresa exportadora de I. guayusa puede generar interés en los (cuasi) monocultivos de dicha especie (Sidali et al. 2016). En este contexto, para ayudar a conciliar la producción alimentaria con la conservación se recomienda:

- Imitar la manera como la agricultura itinerante palía el disturbio por clareo: rodear los potreros de matrices boscosas. Incluir en la forma de cada lote abierto un corredor por donde transiten los insumos y productos, mientras el resto del perímetro esté circundado por los bosques secundarios ya existentes. Eso acelerará la recuperación de los bosques durante los barbechos.

- Aprovechar las estadísticas (e.g., número anual de universitarios, turistas y moradores) para proyectar la dinámica de la demanda alimentaria; ajustando con anticipo el número, las hectáreas, y la cantidad de animales y plantas lucrativas. Ajustar también los tiempos de barbecho por finca para rotar el uso de los terrenos, considerando que hay usos que retardan más que otros la recuperación de los ecosistemas (e.g., Pascarella et al. 2000; este estudio).

- Agregar valor a la agricultura itinerante. Por ejemplo, usándola para turismo responsable -como dijeron muchos de los indígenas entrevistados, y protegiendo a I. guayusa con etiqueta de indicación geográfica, en lugar de favorecer a una sola empresa (Sidali et al. 2016). La indicación geográfica otorga mejores precios a cambio de que la naturaleza y las culturas de todo el territorio permanezcan (Sidali et al. 2016).

\section{CONCLUSIONES}

En los estudios ecológicos, la historia de uso del suelo no debería restiringirse a nombrar para qué se utilizó un lote: es necesario caracterizar qué manipulaciones fueron realizadas y por qué, para inferir mejor cómo eso determina las propiedades que, durante la sucesión secundaria, adquiere un ecosistema. Las historias de uso del suelo aquí documentadas indican que la producción alimentaria y la conservación ambiental en la Amazonía no necesitan estar contrapuestas, porque la cultura de muchas personas incluye comportamientos capaces de permitir que los ecosistemas se regeneren. Para conservar mejor los bosques y la seguridad alimentaria, los científicos, administradores y gobernantes deben aprender de la historia y costumbres de cada sociedad; abrir la mente a las facetas conservacionis- tas de cada cultura, y establecer contextos socioeconómicos que estimulen la conservación de los bosques.

\section{REFERENCIAS}

Albuquerque UP, RFP Lucena, EMFL Neto. 2010. Seleção dos participantes da pesquisa. In Albuquerque UP, RFP de Lucena, LVFC da Cunha eds. Métodos e técnicas na pesquisa etnobiológica e etnoecológica. Recife, Brasil. Nupeea. p. 21-38.

Aweto AO. 2013. Shifting cultivation and secondary succession in the tropics. Oxfordshire, Reino Unido. CABI. 196 p.

Baker TR, OL Phillips, Y Malhi, S Almeida, L Arroyo, A Di Fiore, T Erwin, TJ Killeen, SG Laurance, WF Laurance, SL Lewis, J Lloyd, A Monteagudo, DA Neill, S Patiño, NCA Pittman, JNM Silva, RV Martínez. 2004. Variation in wood density determines spatial patterns in Amazonian forest biomass. Global Change Biology 10(5):545-562. DOI: 10.1111/j.1365-2486.2004.00751.x.

Brienen RJW, OL Phillips, TR Feldpausch, y otros 99 autores. 2015. Long-term decline of the Amazon carbon sink. Nature 519:344-348. DOI: 10.1038/nature14283.

Bürgi M, L Östlund, DJ Mladenoff. 2016. Legacy effects of human land-use: ecosystems as time-lagged systems. Ecosystems 20(1):94-103. DOI 10.1007/s10021-016-0051-6.

Chazdon RL. 2003. Tropical forest recovery: legacies of human impact and natural disturbances. Perspectives in Plant Ecology, Evolution and Systematics 6(1-2):51-71. DOI: 10.1078/1433-8319-00042.

Chazdon RL, MR Guariguata. 2016. Natural regeneration as a tool for large scale forest restoration in the tropics: prospects and challenges. Biotropica 48(6):716-730. DOI: 10.1111/btp.12381.

Connell JH. 1990. "Apparent" versus "real" competition in plants. In Grace JB, D Tillman eds. Perspectives in plant competition. San Diego, Estados Unidos de Norteamérica. Academic Press. p 9-26.

Cuenca ME, O Jadán, K Cueva, C Aguirre. 2014. Carbono y ecuaciones alométricas para grupos de especies y bosques de tierras bajas, Amazonía Ecuatoriana. Cedemaz 4(1):21-31.

DHUA (Del Hierro, Urbanismo y Arquitectura, EC). 2014. Propuesta final de diseño, integración y afectación urbana del proyecto Ikiam con su entorno inmediato: integración a los asentamientos, sistema vial y movilidad, paisajístico y esquemas generales para la dotación de servicios básicos y Conectividad (Producto 1-Lámina A1-01). 1p.

Erazo G, JC Izurieta, P Cronkleton, A Larson, L Putzel. 2014. El uso del pigüe (Piptocoma discolor) por los pequeños productores de Napo, Ecuador: manejo sostenible de una especie pionera para los medios de vida locales. CIFOR Brief 26:1-6. DOI: 10.17528/cifor/004424.

Esquivel-Muelbert A, TR Baker, Dexter KG, y otros 79 autores. 2017. Seasonal drought limits tree species across the Neotropics. Ecography 40(5):618-629. DOI: 10.1111/ ecog.01904.

GAD (Gobierno Autónomo Descentralizado Municipal de Tena, EC). 2016. Datos estadísticos. Consultado 10 ago. 2017. Disponible en http://www.tena.gob.ec/index.php/tena/datos-estadisticos.

Garrido-Pérez EI, P Glasnović. 2014. The search of human-dri- 
ven patterns of global plant diversity: why and how? Brenesia 81-82:96-107.

Garrido-Pérez EI, D Tella-Ruiz, J Rojas-Salvatierra, FR Grefa, L Díaz, K Bonilla, J Grefa. 2017. Human intervention, neutral theory, and the assembly of Amazonian secondary forest communities in Napo, Ecuador. International Journal of Science and Nature 8(3):418-429.

Guariguata MR, R Ostertag. 2001. Neotropical secondary forest succession: changes in structural and funcional characteristics. Forest Ecology and Management 148:185-206. DOI: 10.1016/S0378-1127(00)00535-1.

INPE (Instituto Nacional de Pesquisas Espaciais, BR). 2016. PRODES estima $7.989 \mathrm{~km}^{2}$ de desmatamento por corte raso na Amazonia em 2016. Consultado 10.sep.2017. Disponible en: http://www.inpe.br/noticias/noticia.php?Cod_Noticia $=4344$.

MAGAP (Ministerio de Agricultura, Ganadería, Acuacultura y Pesca, EC). 2011. Mapa de suelos del Ecuador continental (variable taxonomía) escala 1:250.000.

Martin PA, AC Newton, JM Bullock. 2014. Carbon pools recover more quickly than plant biodiversity in tropical secondary forests. Proceedings of the Royal Society of London B: Biological Sciences 280 (1773):20132236. DOI: 10.1098/ rspb.2014.0303.

Pallqui, NC, A Monteagudo, OL Phillips, G López-González, L Cruz, W Galiano, W Chavez, R. Vásquez. 2014. Dinámica, biomasa aérea y composición florística en parcelas permanentes Reserva Nacional Tambopata, Madre de Dios, Perú. Revista Peruana de Biología 21(3):235-242. DOI: 10.15381/rpb.v21i3.10897.

Pascarella JB, TM Aide, MI Serrano, JK Zimmerman. 2000. Land-use history and forest regeneration in the Cayey Mountains, Puerto Rico. Ecosystems 3: 217-228. DOI: 10.1007/ s100210000021.

Pitman NCA, JW Terborg, MR Silman, P Núñez, DA Neill, C Cerón, W Palacios, M Ulestia. 2001. Dominance and distribution of tree species in upper Amazonian Terra Firme forests. Ecology 82(8):2101-2117. DOI: 10.1890/0012-9658(2001)082[2101:DADOTS]2.0.CO;2.

Rivadeneira M. 2004. Breve reseña histórica de la explotación petrolera de la cuenca Oriente. In Baby P, M Rivadeneira, R Barragán eds. La cuenca Oriente: geología y petróleo. Lima, Perú. IFEA- Instituto Francés de Estudios Andinos. p. 205-228.

Sidali KL, PY Morocho, EI Garrido-Pérez. 2016. Food tourism in indigenous settings as a strategy of sustainable development: the case of Ilex guayusa Loes. in the Euadorian Amazon. Sustainability 8(10):967. DOI: 10.3390/ su8100967.

Vitery PV. 2015. Women as creators of biodiverse forests: Ecuadorian Amazon -ancestral system chakra ushun purun. Consultado 19 dic. 2016. Disponible en: https://unfecc6. meta-fusion.com/sb42/events/2015-06-08-10-00/ms-pasvitery-ecuador. 